\title{
A Study on the Reform of the Research Achievements Evaluation System on Regional High-level Universities in the Context of National "Double First-rate" Plan
}

\author{
Fei Li ${ }^{1, a}$, Yi Liu, ${ }^{1, b, *}$, Yan Wen², Xue Ding ${ }^{1}$, Wei Wen ${ }^{1}$ \\ ${ }^{1}$ Yunnan Normal University Kunming, Yunnan, China \\ 'Lanzhou institute of industrial, Gansu, China \\ alifei@ynnu.edu.cn, b397047903@qq.com \\ Corresponding author: Yi Liu
}

Keywords: Double First-rate; Research Achievements; Regional High-level Universities.

\begin{abstract}
As an important part of research achievements management, the evaluation of research achievements is vital to elevate research level and provides important references for first-class discipline construction, teacher appointment, annual assessment, post allowance distribution and professional title grading. With Yunnan Normal University as the research object, based on the actual situation of our university, this paper puts forward a research achievements evaluation system to improve our research achievements management, and promote the construction of first-class disciplines and preponderant disciplines.
\end{abstract}

\section{Introduction}

According to The Overall Plan to Promote the Construction of World-class Universities and World-class Disciplines issued by the State Council (hereinafter referred to as The Plan), "By 2020, China will have a number of world-class universities and a number of world-class disciplines, with several disciplines ranking high on the 'World-class Disciplines' list; by 2030, more universities and disciplines will become world-class, with several universities ranking high on the 'World-class Universities' list, a number of disciplines ranking high on the 'World-class Disciplines' list, and the overall strength of China's higher education being prominently enhanced; by the middle of this century, the number and strength of China's first-class universities and first-class disciplines will be at the forefront of the world, making China a powerful country in higher education." The Plan has received a positive response from and been discussed enthusiastically by the higher education sector, and corresponding studies were immediately carried out [1].

With the acceleration of the "Double First-rate" construction, researches in colleges and universities are continuously deepening and broadening, and the research management has become more and more complicated and comprehensive[2, 4]: First, research scales are large - a project often involves lots of research personnel and huge expenditure, and the funding sources, amounts and categories are diversified; second, different disciplines are increasingly interacting, research results have diverse forms, and cooperative research has become a distinctive feature of contemporary research work. The popularity of research and the speed of innovation have entered a new phase. How to effectively manage the complicated research situation has become an urgent problem for colleges and universities. Therefore, colleges and universities put forward higher requirements on research management, especially on the innovation of research evaluation systems.

At present, the research management departments of various universities are actively exploring their own research evaluation management solutions. It is difficult for us to find an effective and comprehensive evaluation solution from existing internal research evaluation systems to scientifically guide the research management $[3,7]$, the reason is that most internal research evaluation solutions are based on qualitative analysis [5, 6] and supplemented by quantitative analysis, which is inevitably affected by the subjective factors of the researchers while ignoring the differences among different 
research-oriented universities, resulting in absolute evolution standards lacking pertinence. Therefore, developing innovative research evaluation systems and applicable evaluation management solutions is an important work of the research management departments of all universities, including Yunnan Normal University.

\section{The Characteristics of Regional High-level Universities}

It is easy to conclude from the connotation of Regional High-level University that such universities have two salient features: contributing to the society and carrying out original researches. In addition, comprehensive analysis of China's first-class universities and regional high-level universities show that they also have the following common features: First, regional high-level universities have a faculty of reasonable scale and structure, reasonable teacher-student ratio, and rational structure of educational background and professional title, as well as rational age structure and discipline structure. Such universities also have a number of well-known professors and experts, including renowned domestic and foreign academic authorities and scholars. Secondly, disciplines of regional high-level universities are of complete categories and have a higher degree of integration. Such universities attach great importance on inter-disciplinary infiltration and the cultivation of emerging disciplines, and they all have significant characteristics and advantages. At the same time, high-level universities have advanced researches which can make epoch-making achievements or achievements capable of influencing the direction and trend of corresponding fields. Fourthly, such universities have a good education and research environment, as well as active international exchanges and academic activities. Fifthly, regional high-level universities are capable of attracting a large number of education and research talents and recruiting lots of foreign outstanding students; they are the center of scientific, cultural and academic exchanges. Sixthly, regional high level universities have a philosophy and spirit of rich characteristics of the times; they also have free campus culture and enjoy a high academic reputation. Their school running mechanisms are balanced in academic power and administrative power. Seventhly, regional high-level universities play a pivotal role in promoting the political, economic, cultural and social development of the region and the world through the cultivation of talents, or through researches and science and technology service activities. They promote social progress and development, rather than hinder it.

\section{The Need to Evaluate Research Achievements}

As the center of high-level researches, the center to train advanced talents and academic masters, and the center combining science and technology and industry, regional high-level universities play an important role in the "Double First-rate" strategy. The need to evaluate research achievements mainly manifest in the following two aspects: from the micro perspective, research evaluation is an important part of research management; it is vital to elevate research level and provides important references for first-class discipline construction, teacher appointment, annual assessment, post allowance distribution and professional title grading. Scientific evaluation of research achievements is conducive to enhancing the enthusiasm of teachers to carry out researches, and helps to create an innovative academic environment, as well as train and retain talents. From the macro perspective, research evaluation helps the government to examine the rationally and efficiency of the researches in research-oriented universities; the results provide decision-making basis for the government to develop research policies, set priorities and research funding directions ${ }^{[6]}$. Yunnan Normal University has formed a research evaluation mechanism characterized by reunification and quantification, which has played an important role in mobilizing the enthusiasm and creativity of research personnel. However, the contradiction between the research evaluation system and the need to improving the quality and standards of researches is becoming increasingly obvious: problems such as preferring quantity to quality, preferring form to content, preferring short-term results to long-term achievements, as well as unitary evaluation standards and simple evaluation approaches need to be effectively solved. Other issues include inefficient implementation of classified evaluation and lack of 
incentives to facilitate the transformation of scientific and technological achievements, and social services. These problems have greatly affected the sustained and healthy development of researches in universities, so improving the system of research evaluation system is of urgent need.

\section{The Guiding Ideology of Building Research Evaluation Systems:}

The evaluation of researches will be based on the principle of "Encouraging Innovation, Satisfying Needs, Combining Science And Education, Giving Full Play To Special Advantage", with a focus on scientific frontiers and realistic demands, to catalyze significant results. At the same time, universities should establish production-study-research cooperative innovation mechanisms to accelerate innovation, promote the combination of science and education to improve the quality of talent training, pay attention to the quality of science and technology innovation and practical contributions to motivate scientific and technological personnel in different areas and different positions to further fortify their distinguishing features and pursue excellence. The first step is to further promote scientific classification of research evaluation. Universities should develop scientific and rational evaluation criteria with different focuses; these criteria should be aiming at different objects, such as scientific and technological personnel, innovation teams, platform bases and scientific research projects, and cover a wide range of contents, like research integrity and academic atmosphere, scientific dissemination and popularization, mechanism innovation and opening and sharing according to the characteristics of different activities such as basic research, applied research, technology transfer and achievement transformation. The second step is to improve the research evaluation system with quality and innovation as the guide. Universities should attach greater importance on quality instead of quantity, while providing more-generous incentives for major scientific research projects and high-level research achievements; strengthen cooperation with office of educational administration, as well as graduate and personnel management departments; coordinate high-level incentives and scientific research performance; establish a scientific, reasonable and efficient reward distribution system. The third step is to strengthen the macro-coordination. Universities should set clear evaluation requirements on landmark results collectively made by research and innovation teams in accordance with the goal of building a regional high-level university; innovation teams have to be evaluated in a holistic way to highlight their ability to cooperate and solve major scientific and technological problems. Team construction, representative projects and results, mechanisms and culture should all be evaluated. Besides, universities should deepen the reform of distribution systems, taking practical contribution into consideration. The fourth step is to improve research platform bases and other long-term evaluation mechanisms with comprehensive performance and opening and sharing as the focus, and to moderately extend the evaluation cycle of research platform bases according to the types of science and technology activities, as well as academic characteristics. Innovation quality, service contributions, the combination of science and education, talents, mechanisms and culture should all be evaluated; universities should encourage the transformation of new research results, technical services and scientific popularization, while accepting domestic and foreign scholars and encourage the opening and sharing of research facilities.

\section{The Modeling of Research Achievements Evaluation System}

\subsection{The Selection of Research Evaluation Indicators}

Through analyzing the contents of the actual research management in Yunnan Normal University and relevant materials of the internal research evaluation indicator systems of domestic and foreign universities, and conducting expert interviews and consulting sessions to select indicators, This paper put forwards three primary evaluation indicators (research inputs, research outputs and contributions to local economy) (Figure 1), 15 secondary evaluation indicators and over 100 tertiary evaluation indicators. 


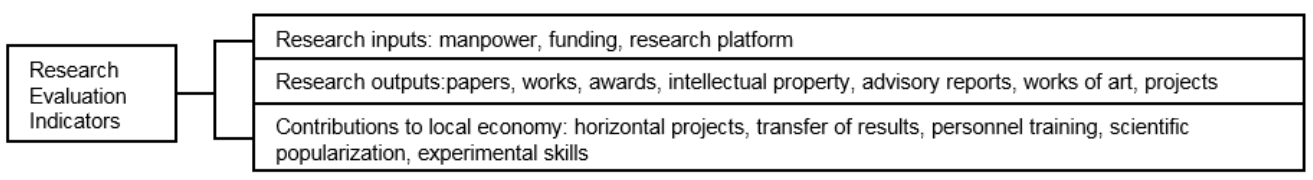

Figure 1. Research Evaluation Indicators

\subsection{The Connotation of Research Evaluation Indicators}

1. Research inputs include three secondary indicators: manpower, funding and research platform. Their descriptions are as follows:

Manpower: manpower is the No.1 resource, the principal participant to perform the research. The number and quality of research personnel are directly related to the research activities; adequate scientific and technological input is the key to improve research ability ${ }^{[2]}$.

Funding: including horizontal funding and vertical funding. Sufficient research funding is a necessary condition for producing quality innovation results, and project funding is an important part of research funds, embodied in the expenditure of various projects in the year evaluated, and the reference is the year-end statistical results.

Research platform: mainly including the state key laboratory or engineering technology research center, the provincial and ministerial state key laboratory or provincial engineering technology research center, the national key disciplines, provincial key disciplines, academicians workstations, think tanks, collaborative centers, center for post-doctoral studies and school-level research institutions. Various research platforms are pools of outstanding talents and incubators of high-level research achievements.

2. Research outputs are the direct and explicit outcomes of research activities. They are important indicators of research evaluation, and can directly reflect the level of research in colleges and universities. Specific indicators are as follows:

The evaluation indicators of papers are shown in Figure 2.

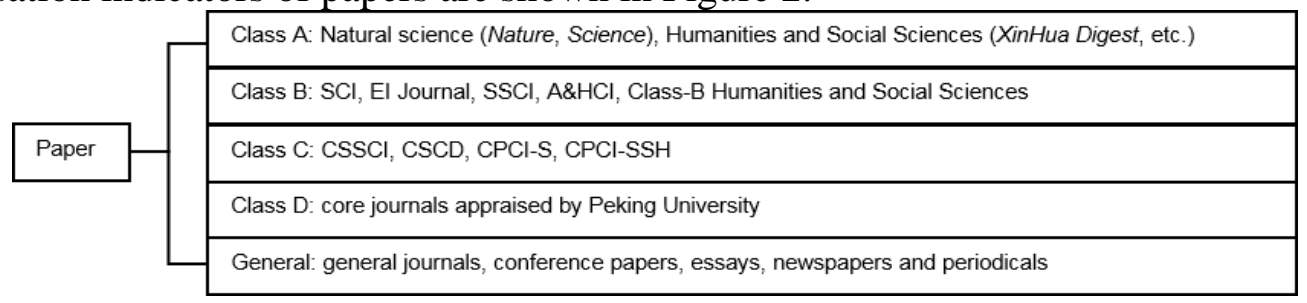

Figure 2. Evaluation Indicators of Papers

Works: here refer to monographs, compilations, translations, etc. published by official publishing departments, including two categories: scientific and technical works and social science works.

Awards: mainly refer to the national, provincial, ministerial and departmental-level research awards issued by party committee and government units.

Intellectual properties are shown in Figure 3.

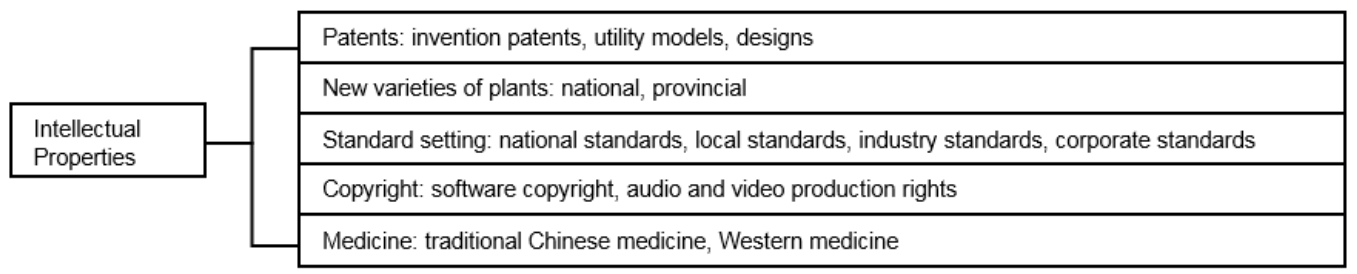

Figure 3. Intellectual Properties Evaluation Indicators System

Consultation reports: mainly refer to the academic reports adopted by the State Council, the National People's Congress, the CPPCC, the ministries and the provincial government.

Art works: mainly refer to the literature, communication, art, music, director, sports and other artistic results publicly published, awarded, exhibited, performed, played or collected.

Technology transfer: technology transfer is a key part of research activities. Only by transforming into products and industries, into real productivity, can research achievements, especially applicable 
research and development results truly become a powerful driving force to promote economic and social development.

Projects: mainly refer to various projects at all levels planned by the government, identified by the school and completed by teaching and administrative staff with the school as the supporting institution (referred to as vertical projects). Evaluation indicators of research projects are shown in Figure 4.

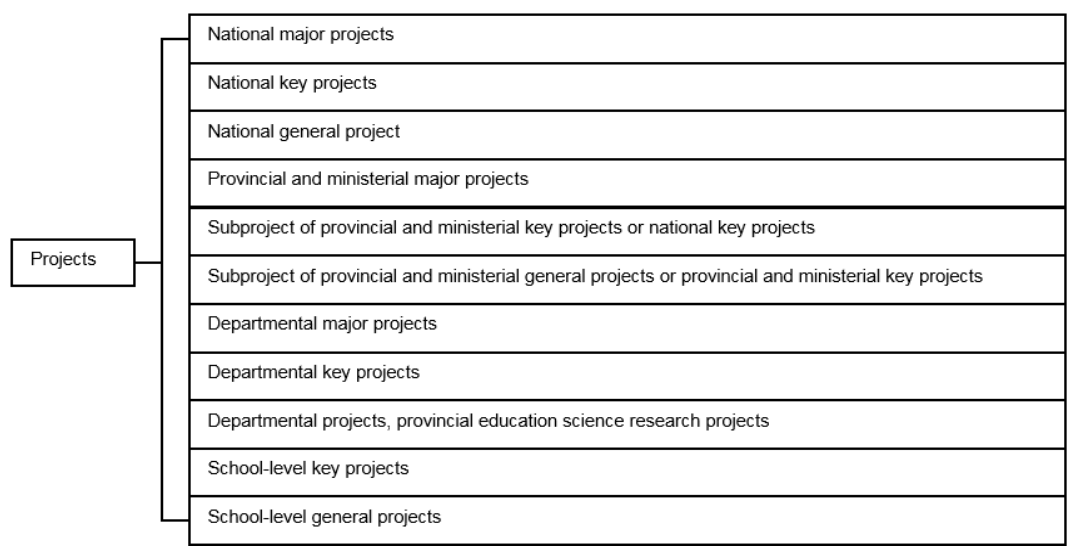

Figure 4. Research Projects Evaluation Indicators System

Contributions to local economy: mainly refer to horizontal projects (projects commissioned by enterprises, local governments and departments), transfer of results, personnel training, scientific popularization, experimental skills

\subsection{Modeling}

$\mathrm{G}=$ Comprehensive evaluation score; $\mathrm{W}=$ Weight $\mathrm{I}=$ Research input; $\mathrm{O}=$ Research output; $\mathrm{S}=$ Contributions to local economy.

$$
G=O \times W_{2}+S \times W_{3}-I \times W_{1}
$$

\section{Summary}

These research evaluation indicators are based on the reality of regional high-level universities in China; they are comprehensive, scientific, oriented and independent. Colleges and universities should develop a complete research evaluation indicators system and define the weight of each indicator according to their actual conditions, to evaluate their research activities in a more objective and impartial way, so as to improve the efficiency of their research management and constantly promote the development of their first-class disciplines.

\section{References}

[1]. Yatao Xue. Introduction to in the course of the construction of "double top" university "adaptation" and "backward" [J]. Journal of higher education, 2016, 15:172-173.

[2]. Yan Jiang. Hunan university scientific research evaluation index system of building research [D]. Hanan: Hunan University, 2007.

[3]. DING Yu, HUANG Yanxia. Brief Review of Research Quality Framework in Australia [J]. Science and technology policy and management, 2008, (5): 29-31.

[4]. TANG Huijun. University scientific research evaluation system and its application research [D]. Hanan: Hunan University, 2006.

[5]. QIAO Wenkai. Research university scientific research performance evaluation index system in our country [D].Gansu: Lanzhou University, 2009.

[6]. SU Xue, WU Guangyin. Preliminary build the evaluation index system of scientific research innovation output [J]. Journal of Intelligence, 2010, 29:138-140.

[7]. LI Su. Scientific research evaluation system research [J]. Pioneering with Science \& Technology Monthly, 2005, 6:13-14. 